\title{
Erratum to: CERN Analysis Preservation: A Novel Digital Library Service to Enable Reusable and Reproducible Research
}

\author{
Xiaoli Chen ${ }^{1,5}$, Sünje Dallmeier-Tiessen ${ }^{1(\bowtie)}$, Anxhela Dani ${ }^{1,2}$, \\ Robin Dasler ${ }^{1}$, Javier Delgado Fernández ${ }^{1,4}$, Pamfilos Fokianos ${ }^{1}$, \\ Patricia Herterich $^{1,3}$, and Tibor Šimko ${ }^{1}$ \\ 1 CERN, Geneva, Switzerland \\ \{xiaoli.chen, sunje.dallmeier-tiessen, anxhela.dani, \\ robin. dasler, javier. delgado. fernandez, \\ pamfilos. fokianos, patricia.herterich, \\ tibor.simko\}@cern.ch \\ 2 Alexander Technological Educational Institute of Thessaloniki, \\ Thessaloniki, Greece \\ 3 Humboldt-Universität zu Berlin, Berlin, Germany \\ ${ }^{4}$ Universidad de Oviedo, Oviedo, Spain \\ ${ }^{5}$ University of Sheffield, Sheffield, UK
}

\section{Erratum to:}

Chapter 27: N. Fuhr et al. (Eds.) Research and Advanced Technology for Digital Libraries DOI: 10.1007/978-3-319-43997-6_27

In an older version of the paper starting on page 347 of this volume, Fig. 2 was incorrect. This has been corrected. 